\title{
VisPublica: uma proposta para aprimorar a transparência de dados públicos
}

\author{
Fernanda C. Ribeiro ${ }^{1}$, Thiago P. Ferreira ${ }^{1}$, Melise M. V. de Paula ${ }^{1}$, Miriam Chaves ${ }^{2}$, \\ Sergio A. Rodrigues ${ }^{3}$, Jano M. de Souza ${ }^{3}$, Ednylton M. Franzosi ${ }^{3,4}$, Luiz F. S. Oliveira ${ }^{4,5}$ \\ ${ }^{1}$ Instituto de Ciências Exatas - Universidade Federal de Itajubá (UNIFEI) \\ Av. BPS, 1303 - Bairro Pinheirinho - 37500-903 - Itajubá - MG - Brasil \\ ${ }^{2}$ Ministério do Planejamento, Orçamento e Gestão \\ Esplanada dos Ministérios - Brasília - DF - Brasil \\ ${ }^{3}$ Programa de Engenharia de Sistemas e Computação - COPPE/UFRJ \\ Caixa Postal: 68511 - 21941-972 - Rio de Janeiro - RJ - Brasil \\ ${ }^{4}$ SERPRO - Serviço Federal de Processamento de Dados - Rio de Janeiro - RJ - Brasil \\ ${ }^{5}$ Instituto de Computação, Universidade Federal Fluminense (UFF)
\{fernandafcrk, sjk.thiago, melisepaula, miriambfc\}@gmail.com, \{sergio, jano\}@cos.ufrj.br, \{ednylton.franzosi, luiz- \\ felipe.oliveira\} aserpro.gov.br
}

\begin{abstract}
Currently, in the governmental context, transparency of information represents a major challenge. This scenario can be analyzed from different perspectives. In the technological aspect, the large volume of data and complex relationships between these data require specific approaches not only in showing but also to provide an easy interpretation of information by citizens. In this article will describe an application developed in the context of Vispublica's Projects, whose objective is to analyze the information visualization techniques to facilitate the interpretation of public data.

Resumo. Atualmente, no contexto governamental, a transparência das informações representa um dos grandes desafios. Este cenário pode ser analisado a partir de diferentes perspectivas. No aspecto tecnológico, o grande volume de dados e a complexidade das relações entre esses dados exigem abordagens específicas não somente para a disponibilização dos dados, mas para a fácil interpretação das informações pelos cidadãos. Neste artigo, será descrita uma aplicação desenvolvida no contexto do projeto VisPublica cujo objetivo é analisar técnicas de Visualização de Informações que facilitem a interpretação de dados públicos.
\end{abstract}

\section{Introdução}

Atualmente, o volume de dados gerados nos diferentes processos organizacionais define um cenário onde é possível observar dois aspectos básicos: a necessidade de definir abordagens eficientes para interpretação dos dados e a exigência crescente de criação de mecanismos que garantam a transparência das informações.

O principal objeto de estudo deste trabalho é a transparência de dados públicos. Contudo, para abordar corretamente este desafio, é essencial associar a este contexto o efeito ativo que a facilidade de uso e a qualidade da informação estabelecem no 
processo de transparência. O cenário pode ainda ser analisado sob outra perspectiva, na qual, a transparência não é o efeito, mas a causa. De acordo com Aló [Aló 2009], por exemplo, a transparência possibilita o acesso, a facilidade de uso, a qualidade do conteúdo e o entendimento das informações que são do interesse de um determinado público.

Este trabalho está inserido no contexto do projeto VisPublica cujo objetivo é estudar técnicas de visualização que facilitem o entendimento das informações, promovendo a transparência dos dados disponibilizados. O que representa o cerne deste projeto é a possibilidade de utilização eficiente das técnicas de Visualização de Informação (InfoVis) para a massificação dos dados públicos. $\mathrm{O}$ projeto está em andamento. Inicialmente, foram realizados vários estudos sobre várias técnicas de visualização disponíveis e uma análise da utilização de algumas destas técnicas na visualização de um conjunto específico de dados públicos por cidadãos de diferentes perfis [Paula et. al 2011].

A partir dos primeiros resultados, foi desenvolvida uma aplicação. O objetivo deste artigo é descrever esta aplicação considerando seus principais componentes funcionais e apresentar um exemplo da utilização do VisPublica na visualização de um conjunto de dados do projeto DadosGov (Portal Brasileiro de Dados Abertos).

O artigo está dividido em cinco seções. Após a introdução, é apresentada a revisão da literatura considerando alguns trabalhos relacionados à transparência, dados governamentais abertos e InfoVis. Na terceira seção, será detalhada a aplicação desenvolvida no contexto do projeto VisPublica. A quarta seção descreve os resultados da análise da utilização do VisPublica a partir de uma possível integração com o projeto DadosGov. Finalmente, a quinta seção apresenta as considerações finais.

\section{Revisão da Literatura}

A transparência, segundo Aló [Aló 2009], pode ter diversos significados em domínios diferentes. No setor público, transparência é um fator vital para o fortalecimento das relações entre o governo e o cidadão [OECD 2011]. Nesta relação, o papel do governo é oferecer informações e serviços pela Web aos cidadãos. O papel da sociedade é utilizar os dados disponibilizados de maneira a aumentar sua participação e interação com as políticas públicas obtendo um melhor entendimento das ações governamentais [EGOVIMPROVING 2009].

Para Vaz et. al [Vaz et. al 2009], a transparência torna os dados governamentais acessíveis a todos e elimina as restrições quanto à tecnologia, legislação e acessibilidade garantindo acesso irrestrito e a utilização dos dados públicos pelos cidadãos. Os dados disponibilizados aos cidadãos são denominados Dados Governamentais Abertos (DGA). Segundo EGOV-IMPROVING [EGOV-IMPROVING 2009], os DGA se referem à publicação dos dados no formato bruto de maneira a tornar as informações do governo acessíveis a todos e permitir a reutilização das mesmas. A publicação dos dados compõe o primeiro de oito princípios estabelecidos pelo grupo Open Government Data (OpenGovData). Segundo o OpenGovData [OpenGovData 2007] o dado governamental será considerado aberto se o mesmo for [Vaz et. al 2011]:

1. Completo: todos os dados públicos estão disponíveis. Dados públicos são dados que não estão sujeitos a limitações válidas de privacidade, segurança ou controle de acesso. 
2. Primário: os dados são publicados tais como são coletados da fonte, com maior nível possível de granularidade e sem agregação ou modificações.

3. Atual: os dados são disponibilizados tão rapidamente quanto necessário a preservação de seu valor.

4. Acessível: os dados são disponibilizados para a mais ampla variedade de usuários e de propósitos.

5. Compreensível por máquina: os dados são razoavelmente estruturados para permitir processamento automatizado.

6. Não discriminatório: os dados são disponibilizados para todos, sem requerimento de registro.

7. Não proprietário: os dados são disponibilizados em formato sobre o qual nenhuma entidade detenha controle exclusivo.

8. Livres de licença: os dados não estão sujeitos a nenhuma restrição de direito autoral, patente, propriedade intelectual ou segredo industrial. Restrições sensatas relacionadas à privacidade, segurança e privilégios de acesso são permitidas.

Já em 2009, Eaves [Eaves 2009] apresentou as três leis dos dados abertos governamentais. Segundo a primeira lei, se o dado não for encontrado e indexado na web, ele não existe. A segunda lei diz que se o dado não estiver aberto e disponível em formato compreensível por máquina, ele não pode ser aproveitado. Por fim, a terceira lei considera que o dado é inútil, se algum framework legal não permitir a replicação dos dados.

De acordo com a segunda lei e o quinto princípio, os dados abertos governamentais devem ser disponibilizados em formatos que permitam o compartilhamento, descoberta, acesso e fácil manipulação pelos usuários desses dados. $\mathrm{O}$ formato recomendado pela W3C (World Wide Web Consortium) para a representação dos dados abertos é o RDF (Resource Description Framework), pois os dados representados neste formato podem ser facilmente combinados com outros dados de múltiplas fontes (Linked Data) [Dietrich et. al 2011].

Segundo Dietrich et. al [Dietrich et. al 2011], o padrão RDF ainda não é muito difundido, mas está entre as tendências das iniciativas de governo aberto. Dentre as iniciativas do Governo Federal Brasileiro para a publicação de dados em formatos reutilizáveis, destaca-se a criação (em 2010) da Política de Disseminação de Dados e Informações que visa estimular e coordenar ações para publicação dos dados [Miranda 2011].

O Governo Federal mantém alguns portais para a divulgação dos dados. No Portal da Transparência, por exemplo, os cidadãos encontram informações sobre despesas, receitas e convênios do governo [Portal Transparência 2011]. Já no Portal Brasileiro de Dados Abertos estão disponíveis dados mantidos pela administração pública, tais como as séries históricas relativas às ações governamentais [DadosGov 2011]. A criação e manutenção de portais cujo objetivo é a divulgação de dados públicos é uma iniciativa do Governo Federal que contribui com a transparência das políticas públicas e permite que a sociedade participe e contribua na tomada de decisão das ações do governo.

Contudo, ainda que os dados estejam disponíveis, a interpretação pode não ser uma atividade fácil de acordo com o volume e a complexidade dos dados. Logo, é 
importante definir abordagens que facilitem o entendimento dos dados. Uma área de estudo que auxilia na compreensão de dados é a Visualização de Informação (InfoVis). A InfoVis é baseada em técnicas que facilitam a extração e o entendimento de informações a partir de representações visuais dos dados [Nascimento e Ferreira 2005].

Keim [Keim 2002] classifica as técnicas de visualização considerando três critérios: tipo de dado a ser visualizado (unidimensional, bidimensional, multidimensional, texto/hipertexto, hierarquias/grafos e algoritmos/software); técnicas de visualização (gráficos convencionais - para representação de um a três atributos, técnicas geométricas, icnográficas, orientadas a pixel e técnicas baseadas em dimensões) e técnicas de interação e distorção utilizadas (projeção, filtragem, zoom, distorção e ligação e seleção - link \& brush).

Segundo Oliveira e Levkowitz [Oliveira e Levkowitz 2003], a classificação das técnicas de visualização pode ser um guia na escolha de uma dessas técnicas para a representação gráfica dos dados. Mas essa escolha não deve limitar-se a um determinado tipo de classificação existente, sendo possível aplicar diferentes técnicas de visualização conforme o objetivo da busca de conhecimento [Yamaguchi 2010].

O VisPublica está alinhado a este contexto. O principal objetivo do projeto é investigar de que forma as técnicas de visualização de informações podem ser aplicadas para facilitar a transparência de dados públicos.

Existem projetos que possuem objetivos semelhantes. O ManyEyes, por exemplo, é um projeto da IBM, criado por Fernanda Viégas e Martin Wattenberg, que disponibiliza aos usuários ferramentas para a criação de visualizações interativas, sendo permitido compartilhá-las com outros usuários [Projeto Many Eyes 2011]. Contudo, um dos grandes diferenciais do VisPublica e o ManyEyes é o público alvo. O VisPublica trabalha com dados governamentais brasileiros e seu público alvo são os órgãos do Governo e cidadãos. Na próxima seção, será descrita a aplicação desenvolvida no projeto VisPublica com objetivo de auxiliar os setores públicos e cidadãos na visualização e interpretação de dados governamentais.

\section{A Aplicação}

Embora o VisPublica seja o nome do projeto com sentido mais amplo, nesta seção, a aplicação desenvolvida será referenciada como VisPublica somente para facilitar o entendimento. As funcionalidades foram definidas e divididas em três seções: catálogo, interação e colaboração.

As funcionalidades do Catálogo representam o resultado do primeiro passo deste estudo e estão associadas à investigação e a documentação das técnicas de InfoVis analisadas e tecnologias disponíveis. Nesta etapa, o objetivo foi analisar a literatura relacionada considerando dois critérios: as técnicas de visualização existentes e as tecnologias disponíveis para cada técnica analisada.

$\mathrm{O}$ termo tecnologia se refere às bibliotecas, APIs (Application Programming Interface - Interface de Programação de Aplicativos) e outros formatos utilizados por diversos fornecedores que disponibilizam gratuitamente versões funcionais das técnicas utilizadas. Vale ressaltar que, embora alguns fornecedores disponibilizem versões proprietárias da implementação de algumas técnicas, os recursos considerados no VisPublica se referem somente às versões gratuitas. 
Para cada técnica/tecnologia analisada, foi elaborada uma documentação contendo: principais características, exemplos, especificações técnicas e um breve tutorial com informações sobre o funcionamento e uso da tecnologia apresentada. Atualmente, está disponível a documentação de 8 técnicas e 10 tecnologias.

Com a finalidade de facilitar a identificação das informações contidas na documentação, foi estabelecido um leiaute padrão para a seção do catálogo. A Figura 1 ilustra a documentação da técnica Gráfico de Colunas utilizando a tecnologia The JIT [JIT 2011]. Inicialmente, a técnica é descrita considerando suas principais características. Após a descrição, segue um exemplo que é sempre apresentado em uma caixa com cantos arredondados e de fundo cinza. No final da página, está descrito o tutorial técnico utilizando um fundo verde claro para diferenciá-lo.

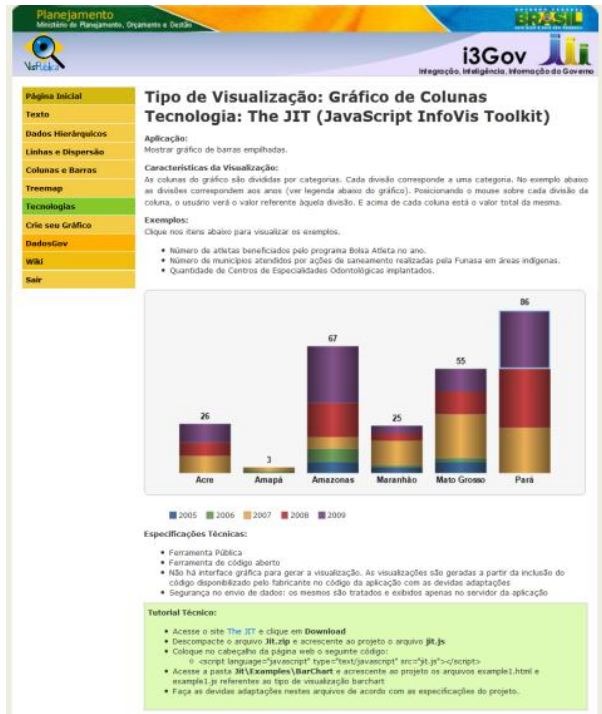

Figura 1. Catálogo - Documentação da técnica Gráfico de Colunas utilizando a tecnologia The JIT.

A seção de interação é acessada a partir do link 'Crie seu Gráfico'. A finalidade é permitir que o usuário utilize as técnicas e tecnologias analisadas para gerar visualizações dos seus dados. A Figura 2 mostra a tela para upload dos dados da técnica Gráfico com Animação (Motion Chart). A princípio, é mostrado um exemplo da técnica para que o usuário veja o resultado da visualização. Após o upload, o exemplo é substituído pela visualização dos dados do usuário.

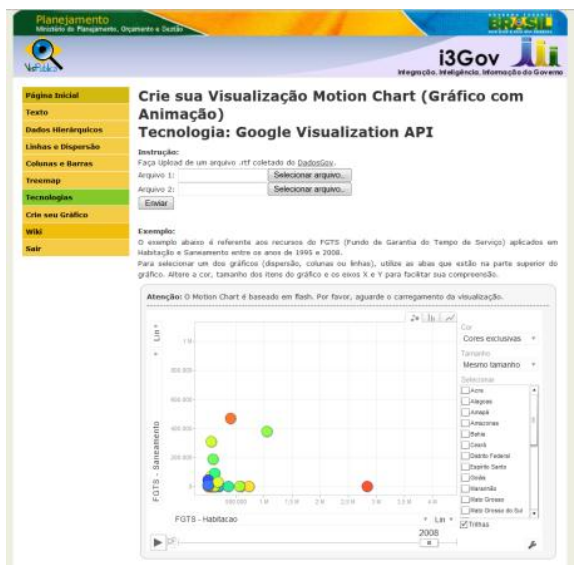

Figura 2. Seção Interação - Crie seu Gráfico - Upload de dados para técnica Motion Chart. 
Por fim, há a seção de colaboração que está associada a um ambiente que permite a edição colaborativa de documentos sobre InfoVis de forma a facilitar a divulgação da informação. Nesta área, os usuários podem compartilhar informações e exemplos sobre técnicas de visualização e tecnologias disponíveis que ainda não constam na seção do catálogo. Por exemplo, a documentação da técnica treemap foi elaborada a partir da página na wiki (Figura 3) que contém exemplos de utilização desta técnica.

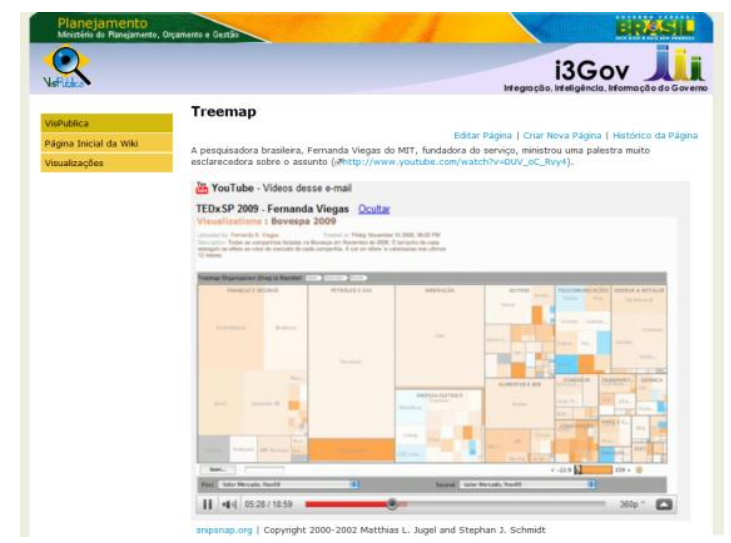

Figura 3. Colaboração - Wiki com exemplo da técnica Treemap.

Na próxima seção, será apresentado um exemplo da utilização do VisPublica a partir da integração com o projeto DadosGov [DadosGov 2011].

\section{Exemplo de Utilização do VisPublica}

Para demonstrar o potencial de utilização do VisPublica, o primeiro passo foi definir quais dados seriam utilizados. Atualmente, está sendo investigada a possibilidade de integração do VisPublica com o Portal Brasileiro de Dados Abertos (DadosGov). DadosGov é "um sistema de informação que agrega e apresenta dados criados ou mantidos pela administração pública" [DadosGov 2011]. Este ambiente é baseado na democratização do acesso à informação e permite que os usuários construam, colaborem e compartilhem aplicativos e visualizações geradas a partir de dados públicos [DadosGov 2011].

No portal, os usuários têm acesso a um catálogo de informações organizado em árvores temáticas com mais de mil séries históricas relativas às ações governamentais fornecidas pelos órgãos públicos. Atualmente, os dados disponibilizados podem ser visualizados em diferentes formatos, tais como: RDF, CSV (Comma Separated Values), XML, JSON (JavaScript Object Notation) e XLS (eXceL Spreadsheet). Além destes formatos para a extração das séries, as mesmas podem ser visualizadas através de tabelas, mapas, gráficos de linha, de setor, de barra e de área [Portal DadosGov 2011].

Investigando as séries disponibilizadas, foi possível perceber que, em alguns casos, os dados se referem a extensos períodos de tempo e a uma considerável quantidade de territórios. Estas características dificultam o entendimento das informações devido, principalmente, ao volume dos dados. Deste modo, a utilização de outras técnicas de visualização poderia facilitar a interpretação destas séries.

Portanto, foram escolhidas quatro séries históricas cada uma pertencendo a um dos quadrantes representados na Figura 4. O eixo Y é o eixo da base territorial, ou seja, indica se as séries históricas possuem dados municipais ("Muitos Territórios") ou 
estaduais ("Poucos Territórios"). O eixo da periodicidade é representado pelo eixo X e associa as séries históricas com períodos anuais e mensais. Séries históricas com dados de 1 a 3 anos estão nos quadrantes de "Poucos Períodos" (2 e 3 da Figura 4). Nos quadrantes de "Muitos Períodos" ( 1 e 4 da Figura 4) estão as séries com periodicidade maior que 3 anos ou periodicidade mensal.

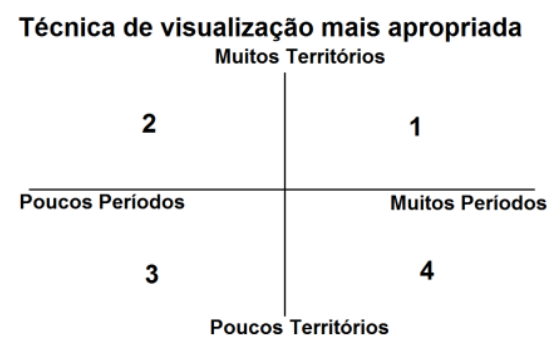

Figura 4. Eixo Período x Território.

Para cada quadrante, foi selecionada uma série histórica:

- Quadrante 1: "Empregos registrados anualmente desde 2002" [Série1 2011].

- Quadrante 2: "Valor financeiro aplicado no Pronaf Reforma Agrária (2002 a 2010)" [Série2 2011].

- Quadrante 3: "Quantidade de Matrículas Ofertadas - Campi Avançados (2009 a 2010)" [Série3 2011].

- Quadrante 4: "Recursos do FGTS aplicados em Habitação (1995 a 2009” [Série4 2011].

Depois de selecionada as séries, foi realizada uma análise das técnicas de visualização disponíveis de forma a identificar a mais adequada para cada conjunto de dados. Segundo Yamaguchi [Yamaguchi 2010], um dos fatores a ser considerado para a escolha da técnica de visualização mais apropriada é o tipo de dado manipulado. Logo, para iniciar a análise do potencial do VisPublica, as séries históricas foram classificadas de acordo com a dimensão dos dados, ou seja, verificou-se se as séries possuem dados uni, bi, tri ou multidimensionais.

De acordo com a estrutura do arquivo XML das séries históricas com periodicidade anual [Série2 2011], os dados podem ser classificados como tridimensionais, pois os mesmos possuem 3 atributos: valor, ano e codibge (código do estado ou município de acordo com o IBGE - Instituto Brasileiro de Geografia e Estatística). Para as séries históricas com periodicidade mensal que apresentam dados com 4 atributos (ano, mês, codibge e valor), os dados foram classificados como multidimensionais. Além da dimensão, as técnicas de visualização também podem ser avaliadas segundo o volume de dados. Yamaguchi [Yamaguchi 2010] ressalta que as técnicas estão sujeitas a algumas limitações, como é o caso da quantidade de dados que cada técnica pode exibir. Logo, o volume de dados deve ser considerado durante a avaliação e escolha da técnica mais apropriada.

As séries do quadrante 1 (Figura 4) possuem um grande volume de dados ("Muitos Períodos" e "Muitos Territórios"). Para dados assim caracterizados, a técnica selecionada é o motion chart (gráfico com animação), pois essa técnica permite a visualização de extensos períodos de tempo. O motion chart da API do Google [MotionChart 2011] possui três tipos de gráficos: gráfico de bolhas, gráfico de colunas e gráfico de linhas. Para mostrar o potencial desta técnica para a Série 1 [Série1 2011] será utilizado o gráfico de colunas. 
A visualização disponível no Portal DadosGov está representada na Figura 6. Nesta visualização, é possível analisar somente o total do período especificado (2002 a 2009). Na figura 5, os mesmos dados são visualizados utilizando a técnica Motion Chart. A principal diferença entre as suas alternativas apresentadas se refere à possibilidade de interação do usuário com a representação. O Motion Chart proporciona uma visualização dinâmica dos dados o que aumenta a significância nas informações representadas. O botão play da barra de animação ilustrada na Figura 5 é responsável por reproduzir a visualização dinâmica. Desta forma, o usuário pode ver a relação dos dados ano a ano. Na Figura 5a, o ano visualizado é 2002. Além disso, no Motion Chart, há também o recurso de zoom, que permite ao usuário focalizar determinada área do gráfico (Figura 5b) aumentando o campo de visão dos dados. Este recurso facilita a visualização de gráficos com muitas colunas.

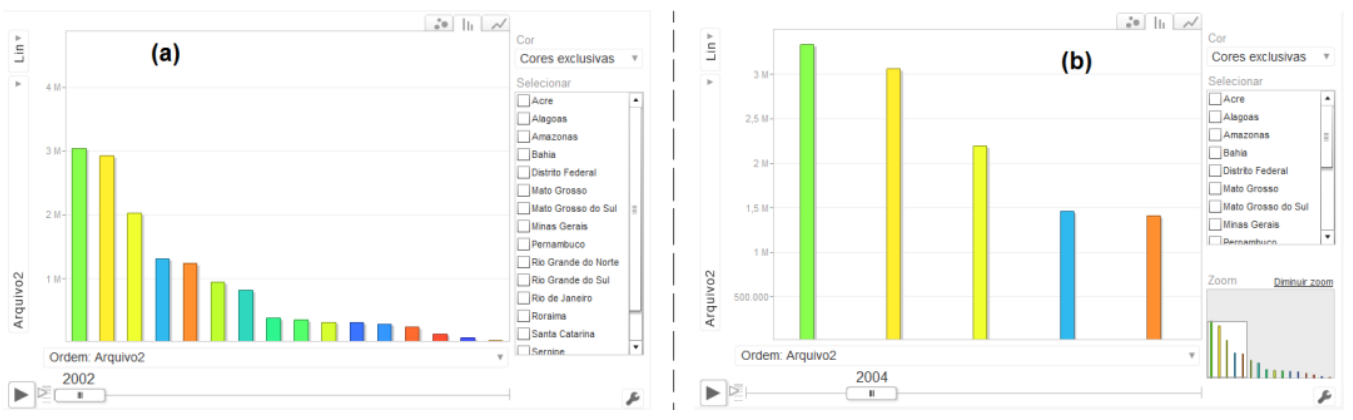

Figura 5. (a) Gráfico de Barras - Motion Chart (b) Zoom nos dados.

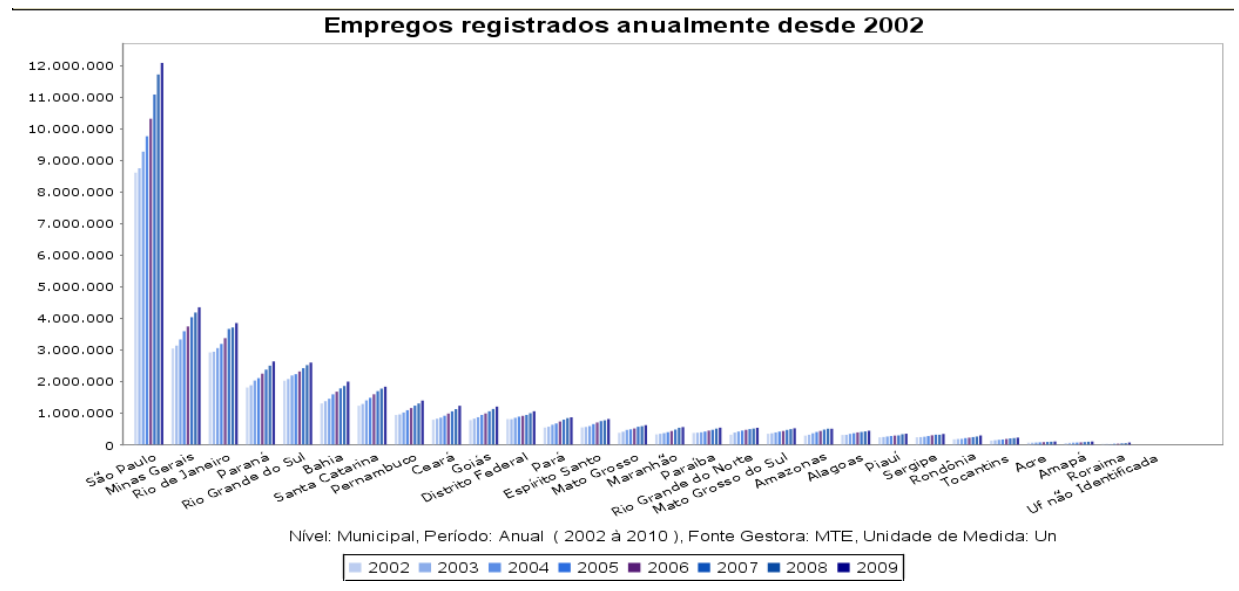

Figura 6. Gráfico de Barras - DadosGov.

Para séries pertencentes ao quadrante 2, o treemap foi a técnica escolhida para representar os dados. O treemap da Figura 7a representa os dados da Série 2 [Série2 2011] que possuem periodicidade de 1 ano e base territorial municipal. Comparando o treemap (Figura 7a) com o Gráfico de Colunas disponível no Portal DadosGov [Portal DadosGov 2011] (Figura 7b), nota-se que no Gráfico de Colunas não são apresentados os dados de cada município, mas o somatório destes por estado. Logo esse gráfico não proporciona maior significância nas informações apresentadas, pois não é possível saber qual o valor repassado a cada município.

No treemap, os municípios são representados pelos retângulos e categorizados por estado e região. Esta divisão permite que o usuário visualize tanto os dados municipais, quanto estaduais e regionais. Além disso, o treemap possui recursos interativos que alteram o foco da visualização. Por exemplo, para o usuário descobrir 
qual o valor repassado a cada município paulista, basta passar o mouse sobre o retângulo correspondente.
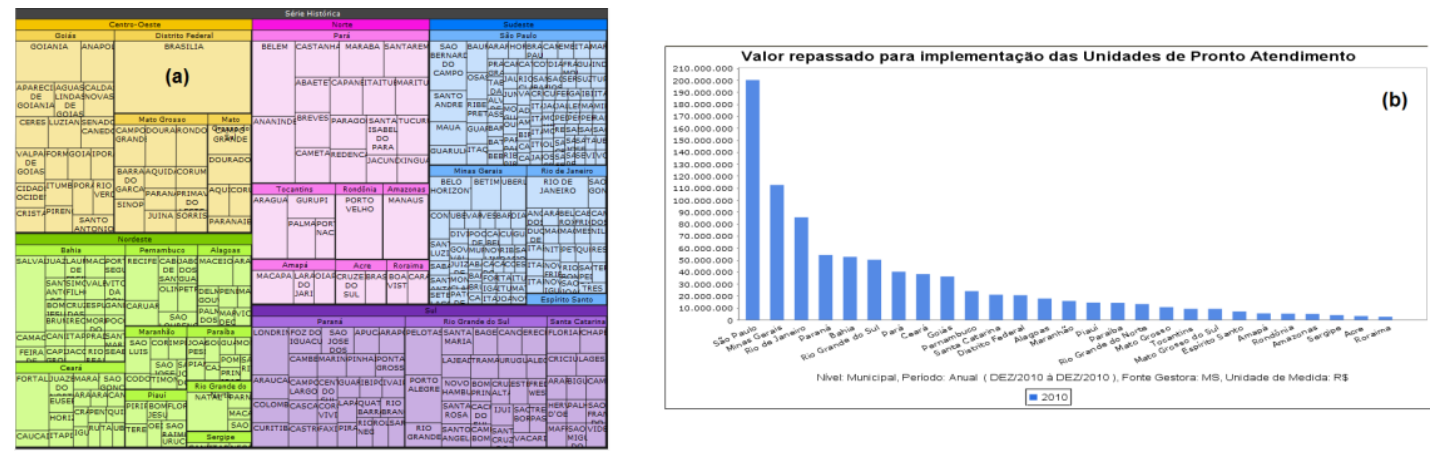

Figura 7. (a) Treemap - VisPublica (b). Gráfico de Colunas - DadosGov.

No quadrante 3, estão as séries históricas caracterizadas com "Poucos Territórios" (11 estados) e "Poucos Períodos" (2009 a 2010) [Série3 2011]. Segundo Yamaguchi [Yamaguchi 2010], para séries assim caracterizadas os gráficos estatísticos (gráfico de barras, colunas, linha, pizza) são ideais para representar os dados, pois o pequeno volume de dados e a baixa dimensionalidade proporcionam uma visualização clara ao usuário.

No VisPublica o gráfico da Figura 8a é construído a partir da tecnologia Highcharts [Highcharts 2011] e possui uma opção de interação (seleção). Por exemplo, o usuário ao clicar em um item da legenda, o mesmo é retirado (Figura 9) possibilitando modificações na visualização que contribuam para o entendimento da informação representada (Figura 8b). Para retornar à visualização original (Figura 8a), o usuário deve clicar nos itens ocultos pela legenda.
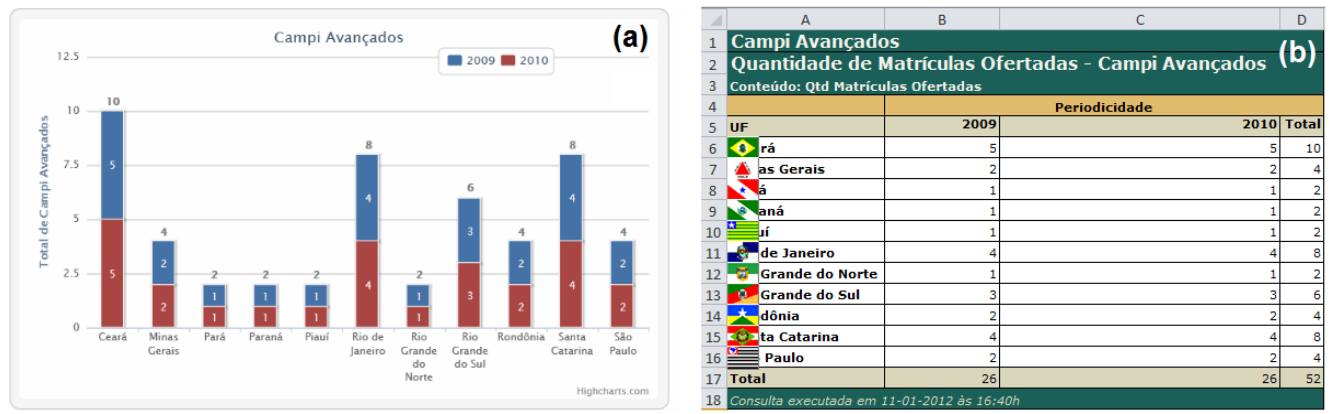

Figura 8. (a) Gráfico de Colunas (b) Arquivo XLS.

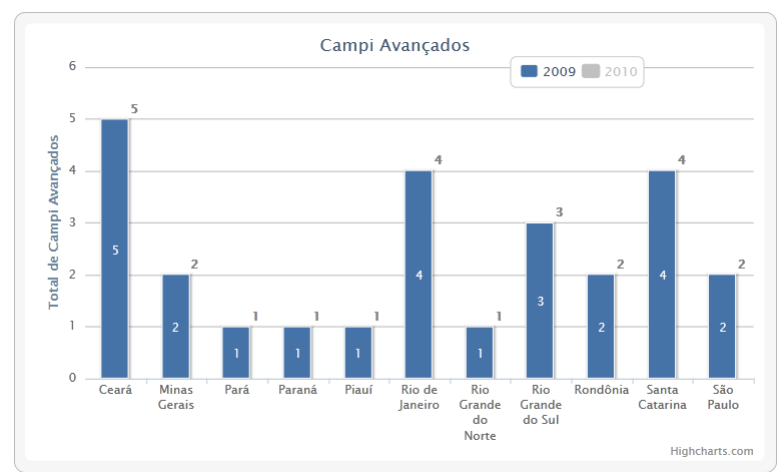

Figura 9. Gráfico de Colunas sem o item '2010' da legenda. 
Para as séries do quadrante 4 (Figura 4) que são caracterizadas com "Poucos Territórios" e "Muitos Períodos", a técnica selecionada também foi o Motion Chart. Dos três tipos de gráficos que o Motion Chart apresenta, o gráfico de bolhas foi o escolhido para demonstrar o potencial para mostrar o potencial da técnica. Para isso serão utilizadas duas séries históricas do quadrante $4 \mathrm{com}$ a finalidade de compará-las ao longo do tempo. No eixo X estão os dados referentes à Série 4 [Série4 2011] e no eixo Y dados da Série 5 [Série5 2011] (Figura 10a).

Os estados brasileiros serão representados pelas bolhas e diferenciados pela cor. Por exemplo, a bolha vermelha representa o estado de São Paulo (Figura 10a). Abaixo do eixo X fica a barra de animação para controlar o intervalo de tempo (no exemplo, de 1995 a 2009). Há também a opção de selecionar um ou mais estados e visualizar a trajetória dos dados referentes a este estado ao longo do tempo (Figura 10b).
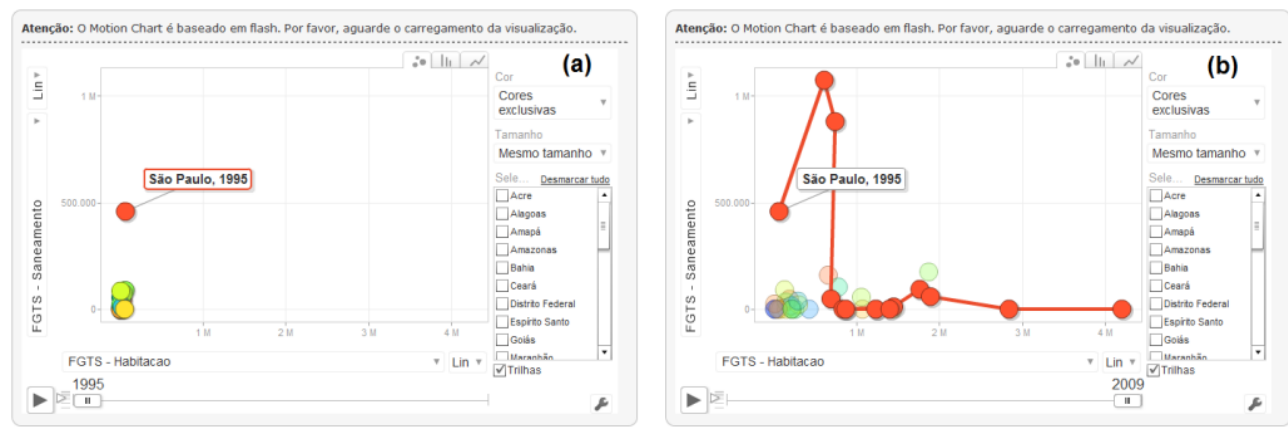

Figura 10. (a) Motion Chart (b) Trajetória dos dados referentes à SP

\section{Considerações Finais}

O VisPublica é um projeto, em desenvolvimento, que estuda técnicas de visualização de dados públicos de forma a aprimorar a transparência das informações. Um dos resultados deste projeto é a aplicação descrita neste artigo. Nesta fase do projeto, é possível fazer algumas considerações.

A utilização das técnicas de visualização requer um esforço considerável de análise para garantir a adequação da técnica ao dado a ser visualizado. Por exemplo, a série selecionada do quadrante 1 foi inicialmente visualizada utilizando a técnica treemap. Ainda que a técnica seja proposta na literatura para visualizar grandes volumes de dados, foi possível verificar que para grandes períodos de tempo, a técnica não foi adequada.

Embora, já existam várias tecnologias disponíveis, algumas apresentam limitações que precisam ser solucionadas. Por exemplo, a técnica nuvem de tags (tag cloud) pode ser implementada usando a API do Google [Tag Cloud 2011]. Contudo, não é possível retirar da visualização as palavras comuns, tais como: preposições, artigos, pronomes e conjunções. No VisPublica, esta limitação foi solucionada alterando o código da API de forma que o usuário possa retirar da visualização as palavras comuns e também qualquer palavra que ele não deseja que apareça na tag cloud do texto.

A utilização das técnicas do VisPublica para visualizar, por exemplo, as séries históricas do DadosGov mostra o potencial do VisPublica como ferramenta para auxiliar na visualização de dados públicos. A integração entre o VisPublica e DadosGov representa o próximo passo do projeto. O objetivo é permitir que os dados 
disponibilizados no portal possam ser visualizados utilizando diferentes técnicas já disponíveis no VisPublica.

\section{Referências Bibliográficas}

Aló, C. C. (2009) "Uma abordagem para transparência em processos organizacionais utilizando aspectos". Dissertação de Doutorado em Informática. Pontifícia Universidade Católica do Rio de Janeiro, Rio de Janeiro.

DadosGov. (2011) "O que é o dados.gov.br". Disponível em: $<$ http://beta.dados.gov.br/sobre.html>. Acessado em 27 de dez. 2011.

Dietrich, D., Gray, J., McNamara, T., Poikola, A., Pollock, R., Tait, J. e Zijlstra, T. (2011) "The Open Data Manual". Disponível em: <http://opendatamanual.org>. Acesso em 28 de dez. 2011.

Eaves, D. (2009) "The Three Laws of Open Government Data". Disponível em: $<$ http://eaves.ca/2009/09/30/three-law-of-open-government-data/>. Acesso em $27 \mathrm{de}$ dez. 2011.

EGOV-IMPROVING. (2009) "Improving Acess to Government through Better Use to the Web". Editado por S. Accar, J. Alonso e K. Novak, W3C Group Note.

Highcharts. (2011) "Highcharts JS. Interactive JavaScript charts for your web projects". Disponível em <http://www.highcharts.com/>. Acesso em 06 de janeiro de 2012.

JIT. (2011) "JavaScript InfoVis Toolkit. Create Interactive Data Visualizations for the Web". Disponível em: < http://thejit.org/>. Acesso em 06 de jan. 2012.

Keim, D. A. (2002) "Information visualization and visual data mining". IEEE Transactions on Visualization and Computer Graphics, 8(1):18.

Miranda, C. M. C. (2011) “A Disseminação de Dados Governamentais como Serviço Público - Os Dados Abertos Governamentais e a Experiência Brasileira", In: CONSEGI 2011 IV Congresso Internacional Software Livre e Comércio Eletrônico: Dados Abertos para a Democracia na Era Digital. Brasília, Fundação Alexandre de Gusmão, 2011, p. 63.

MotionChart. (2011) "Visualization: Motion Chart". Disponível em: $<$ http://code.google.com/intl/pt-

BR/apis/chart/interactive/docs/gallery/motionchart.html>. Acesso em 06 de jan. 2012.

Nascimento, H. A. D. do e Ferreira, C. B. R. (2005) "Visualização de Informações uma abordagem prática", In: XXV Congresso da Sociedade Brasileira de Computação. A Universidade da Computação: um agente de inovação e conhecimento. São Leopoldo, RS. P. 1262 - 1312.

OECD - Organization for Economic Co-operation and Development. (2011). Disponível em: <http://www.oecd.org>. Acesso em 02 de jan. 2012.

Oliveira, M. C. F. de e Levkowitz, H. (2003) "From visual data exploration to visual data mining: A survey". IEEE Transactions on Visualization and Computer Graphics, 9(3):378394.

OpenGovData. (2007) "8 Principles of Open Government Data". Disponível em: $<$ http://www.opengovdata.org/home/8principles $>$. Acesso em 27 de dez. 2011. 
Paula, M. M. V. de, Ribeiro, F. C., Chaves, M., Rodrigues, S. A. e Souza, J. M. de. (2011) "A Visualização de Informação e a Transparência de Dados Públicos", In: Simpósio Brasileiro de Sistemas de Informação. Salvador, Bahia.

Portal DadosGov. (2011) "DadosGov". Disponível em: $<$ https://i3gov.planejamento.gov.br/>. Acesso em 04 de jan. 2012.

Portal Transparência. (2011) "O Portal da Transparência do Governo Federal". Disponível em <http://www.portaltransparencia.gov.br/>. Acesso em 28 de dez. 2012.

Projeto Many Eyes, 2011. "Many Eyes" Disponível em: $<\mathrm{http}$ //fernandaviegas.com/democratizing_viz.html>. Acesso em 10 de jan. 2012.

Série1. (2011) "Empregos registrados anualmente desde 2002". Disponível em: $<$ https://i3gov.planejamento.gov.br/dadosgov/xml/serie/285.xml $>$. Acesso em $06 \mathrm{de}$ jan. 2011.

Série2. (2011) "Valor financeiro aplicado no Pronaf Reforma Agrária". Disponível em: $<$ https://i3gov.planejamento.gov.br/dadosgov/xml/serie/480.xml>. Acesso em 06 de jan. 2011.

Série3. (2011) "Quantidade de Matrículas Ofertadas - Campi Avançados”. Disponível em: $<$ https://i3gov.planejamento.gov.br/dadosgov/xml/serie/150.xml $>$. Acesso em 06 de jan. 2011.

Série4. (2011) "Recursos do FGTS aplicados em Habitação". Disponível em: $<$ https://i3gov.planejamento.gov.br/dadosgov/xml/serie/228.xml>. Acesso em $06 \mathrm{de}$ jan. 2011.

Série5. (2011) "Recursos do FGTS aplicados em Saneamento". Disponível em: $<$ https://i3gov.planejamento.gov.br/dadosgov/xml/serie/229.xml>. Acesso em $06 \mathrm{de}$ jan. 2011.

Tag Cloud. (2011) "WordCloud - Sample". Disponível em: <http://visapigadgets.googlecode.com/svn/trunk/wordcloud/doc.html>. Acesso em 06 de jan. 2011.

Vaz, J. C., Ribeiro, M. M. e Matheus, R. (2011) "Dados governamentais abertos e seus impactos sobre os conceitos e práticas de transparência no Brasil". Disponível em: $<w w w . p o r t a l s e e r . u f b a . b r / i n d e x . p h p / p p g a u / a r t i c l e / v i e w / 5111 / 3700>$. Acesso em 27 de dez. 2011.

Yamaguchi, J. K. (2010) "Diretrizes para a escolha de técnicas de visualização aplicadas ao processo de extração do conhecimento". Dissertação de Mestrado em Ciência da Computação. Universidade Estadual de Maringá, Maringá. 